\title{
Experimental Research on Modulus of Elasticity with the Ultrasound Measurement Method
}

\author{
Yanxia Wang, Hai Yun*, Tiantian Sun, Qinghui Shang, Xiaomin Ma \\ School of Traffic and Vehicle Engineering, Shandong University of Technology, \\ 255049, ZiBo, china. \\ E-mail: wangyx200@163.com
}

\begin{abstract}
Keywords: ultrasound wave; measurement method; modulus of elasticity; engineering mechanic experimental; engineering mechanic teaching.
\end{abstract}

\begin{abstract}
Modulus of elasticity is an important mechanical parameter that characterizes elastic property of materials. In the process of engineering mechanic learning, modulus of elasticity of materials is mainly measured by electrical measurement method. But electrical measurement method requires students being trained for experimental operation that takes long experimental time and uses one strain gauge for one test making high costs of instruments. Consequently, ultrasound measurement method is put forward to measure modulus of elasticity of materials. In this paper, ultrasound measurement method and electrical measurement method are used to measure modulus of elasticity of Mullite ceramic. Experimental results show that modulus of elasticity measured in ultrasound measurement method tests is $27.7 \mathrm{Gpa}$ and the measured value in electrical measurement method tests is 28.16Gpa. Comparing the two groups of experimental data,it is found that data obtained by ultrasound measurement method is compact while data obtained by electrical measurement method is dispersed and the average value of two groups variate within $5 \%$. As a consequence,ultrasound measurement method can be used to measure modulus of elasticity of ceramic materials. This method broadens students' knowledge horizon and improves students' experiment analytical abilities ,being suited for promotion in college engineering mechanical curriculum with fewer classes and more students participate in experiments.
\end{abstract}

\section{Introduction}

Modulus of elasticity is an important mechanical parameter that characterizes elastic property of materials and is also a significant parameter used in theoretical analysis and designing calculation of structures[1,2]. In the process of engineering mechanic learning, modulus of elasticity of materials is mainly measured by electrical measurement method. However, electrical measurement method requires students being trained for experimental operation that takes long experimental time and uses one strain gauge for one test making high costs of instruments.

This paper measures modulus of elasticity of materials by ultrasound measurement method. This method can be used to calculate property indicators of materials such as shear modulus、Yong's modulus and Poisson ratio by measuring density v velocity of transverse wave and longitudinal wave in materials. This method has benefits such as no use of strain gauge,low costs, short cycle and multi-point measurement, thus it is suitable for multi-person experiment. In addition, this method can broaden students' knowledge horizon and cultivate independent thinking ability and practical ability.

\section{EXPERIMENT METHOD}

Experimental theory. The wave equation of sonic propagation in elastic solid medium is obtained according to the constitutive equation and continuity equation of isotropic materials combined with particle motion equation[3,4]. 


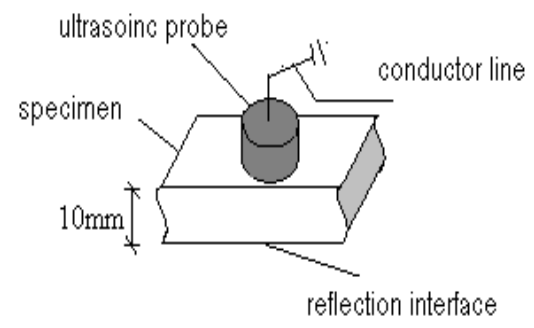

$\rho$ is density of material, $\lambda$ is lame constant, $G$ is shear modulus, $\xi 、 \eta 、 \zeta$ are component functions of particle displacement in three coordinate axis; $\Delta$ is relative volume deformation ; $\nabla^{2}$ is Laplasse operator:

It is supposed that vibration displacement $(\xi=\xi(\mathrm{x}, \mathrm{t}), \xi=\zeta=0)$ in $X$ axis is the only particle displacement in infinite elastic medium and volume force $X=Y=Z=0$. Substitute it into equation (1) it is obtained that

$$
\left.\begin{array}{l}
\frac{\partial^{2} \xi}{\partial t^{2}}=c_{L}{ }^{2} \frac{\partial^{2} \xi}{\partial x^{2}} \\
c_{L}=\sqrt{\frac{\lambda+2 G}{\rho}}
\end{array}\right\}
$$

According to the relationship of $\lambda$, shear modulus $G$, modulus of elasticity $E$, poisson ratio ${ }^{\mu}$, the velocity of longitudinal wave in infinite homogeneous medium will be gotten.

Similarly, if $\eta=\eta(\mathrm{x}, \mathrm{t})$ the velocity of transverse wave in infinite homogeneous medium is obtained as follows:

$$
\left.\begin{array}{l}
c_{T}=\sqrt{\frac{G}{\rho}} \\
c_{T}=\sqrt{\frac{E}{2 \rho(1+\mu)}}
\end{array}\right\}
$$

Comparing formula (2) with (3), it indicates that the velocity of propagation of the same sonic wave in solid medium is determined by density and modulus of elasticity. Furthermore, the elastic deform of medium caused by different waveform spread in medium as well as the velocity of propagation is different, velocity of transverse wave is less than longitudinal wave.

Inverse elasticity by formula of transverse wave velocity and longitudinal wave velocity as follows:

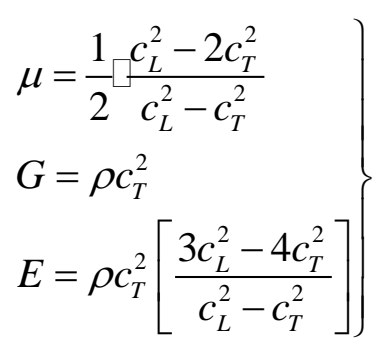

$C_{T}$ is velocity of transverse wave, ${ }^{C_{L}}$ is velocity of longitudinal wave, $E$ is modulus of elasticity, $\rho$ is density of specimen.

It is shown in formula (4) that elastic parameters such as shear modulus, Young's modulus, and Poisson's ratio of materials can be calculated by the measured value of density, velocity of transverse and longitudinal wave. In addition, the properties of materials can be evaluated indirectly by measuring sonic velocity of materials because elastic coefficient is function of sonic velocity[5,6].

Manufacture of specimen and instrument operation. The shape of experimental specimens is rectangle, its size is $10 \mathrm{~mm} \times 20 \mathrm{~mm} \times 100 \mathrm{~mm}$. Instruments used in ultrasound test include OLMPUS 
pulse generator,preamplifier, numerical oscilloscope, shear wave probe,coupling agent, vernier caliper,electronic balance instrument. Selected 5 specimens are manufactured by dry pressing sintering method,each one is labeled 1,2,3,4,5 respectively.

Experimental process. Two different sending probes that send transverse wave and longitudinal wave respectively are placed on one of the two parallel surfaces with coupling agent covered on the probe surface so that probe can receive ultrasound reflected from bottom interface.

Mass and length measurement of specimens must be taken before the ultrasound propagation time measurement. Furthermore, frequency and resistance of pulse generator need to be set at appropriate interval avoiding overlap of transmitted wave and reflected wave.

The instrument work principle[7-11]: first, pulse generator send pulse signal to two different sending probes that send transverse wave and longitudinal wave respectively and send signal to numerical oscilloscope; Second, ultrasound transfer to specimens through coupling agent and reflect multiple times in specimens; Third, each echo signal that reaches probe will be received and transferred to amplifier so as to send signal to numerical oscilloscope. At last, current sent pulse signal and multiple echo signal is shown on numerical oscilloscope and once the time difference is calculated propagation time will be obtained. Wave velocity measurement is shown in Fig. (1).

In short, the working process is that pulse signal generated by pulse generator is conversed into high-frequency mechanical energy and then transferred to specimens through coupling agent,propagate, reflect, refract, attenuate to death and finally the propagation time is shown on oscilloscope.

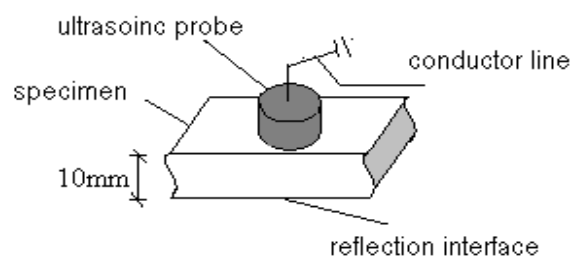

Fig. (1). Ultrasonic wave velocity measurement schematic diagram

\section{Experimental Data and Analytical Results}

Experimental data. Modulus of elasticity of ceramic measured by electrical measurement method and ultrasound measurement method are listed as Table 1.

Table 1 Testing results obtained by electrical method and ultrasound method.

\begin{tabular}{|c|c|c|c|c|c|c|}
\hline & 1 & 2 & 3 & 4 & 5 & average value \\
\hline velocity of longitudinal wave (m/s) & 3995 & 3978 & 4009 & 3966 & 3985 & 3990 \\
\hline velocity of transverse wave (m/s) & 2489 & 2488 & 2487 & 2487 & 2488 & 2493 \\
\hline $\begin{array}{c}\text { Modulus of elasticity obtained by } \\
\text { ultrasound method (GPa) }\end{array}$ & 26.85 & 26.74 & 26.92 & 26.65 & 26.78 & 27.7 \\
\hline $\begin{array}{c}\text { Modulus of elasticity obtained by } \\
\text { electrical method (GPa) }\end{array}$ & 27.70 & 28.31 & 28.35 & 27.37 & 29.07 & 28.16 \\
\hline
\end{tabular}

Analysis of experimental results. Conclusions obtained from Table 1 are as follows:

(1) Data obtained by ultrasound measurement method are compact while data obtained by normal method are dispersed, and the value variate within $5 \%$.

(2) The velocity of longitudinal wave propagation range from $3990 \mathrm{~m} / \mathrm{s}$ to $4010 \mathrm{~m} / \mathrm{s}$ and the variation of sonic velocity is a result of uneven distribution of material in the pressure bonding process of ceramic specimens. There are tiny displacements in ceramic structure so that waves are severely scattered restricting the testing size of ceramic. So appropriate detecting frequency should be chosen in experiments.

(3) Under the same temperature, measured velocity of transverse wave and longitudinal wave in steel is about $2900 \mathrm{~m} / \mathrm{s}$ and $5200 \mathrm{~m} / \mathrm{s}$ while that velocity in dry pressing ceramics is about $2480 \mathrm{~m} / \mathrm{s}$ and $4000 \mathrm{~m} / \mathrm{s}$. Modulus of elasticity can be indicated by comparation of velocities of transverse wave and longitudinal wave in different specimens. 
(4) Values measured by ultrasound method and electrical method indicate that ultrasound measurement method can be applied to measure modulus of elasticity , Poisson ratio, shear modulus and other elastic parameters.

\section{Summary}

The college engineering mechanical experiment lacks acoustics and optical knowledge because of limited class hours. This paper presents ultrasound method for measurement of elastic properties of materials, which extends students' knowledge and promote the passion and enthusiasm. It is required that each student measure experimental data on different position in case of mutual copy. This method help students broaden knowledge horizon and consolidate theoretical knowledge. Viewing from teaching effects, experimental period and costs, this method is suitable for promotion in college engineering mechanic teaching with more students participating experiments and fewer class hours.

\section{References}

[1]Hongwen Liu, Material mechanic[M]. Beijing: higher education press, 2011.

[2]Shiyuan Zhou, Lei Chen, Dingguo Xiao, Chunguang $\mathrm{Xu}$. The method of improving measurement accuracy of sonic velocity in modulus of elasticity measurement test[J]. Technology of sensor, 2005, 24(4): p42 44.

[3]Zhilun Xu. Elasticity mechanic[M]. Beijing: Higher education press, 2010.

[4]Zuoyong He, Yufang Zhao. Basical theory of sonic[M]. Beijing: Defense industry press, 1981.

[5]Jiankai Hu, Qianlin Zhang. Principals and methods of ultrasound test[M],Anhui: University of technology of China press, 1993.

[6]Fangcheng He, Zhaobin Gong. Ultrasound measurement technology and its application in material evaluation[J]. Material engineering, (8) : 32 34.

[7]Yifang Chen, Jiajun Zhang . Research on ultrasound detection of modulus of elasticity[J]. Nondestructive detection, 1997,19 (9) : 241 244 .

[8]Yongchang Chen - Ultrasonic nondestructive evaluation of thermal fatigue damage[D]. Dalian: Dalian university of technology press, 2007

[9]Yinlei Zhou, Mingshui Zhao, Qian Liang. Measurement of modulus of elasticity of roll by ultrasound method[J]. Nondestructive detection, 1994,16 (12) : 347 350.

[10]Paul M, Albert S B, Robert E G . Nondestructive Testing Handbook - Ultrasonic Testing[M], 1996: 224

[11]Yiwei Shi. Ultrasound detection[M]. Beijing: Mechanical industry press. 2005. 